\title{
Release of endogenous chondroitin sulfate and heparin as consequence of dysregulated proteolysis in COVID-19
}

Marco Ruggiero ( $\nabla$ marco.ruggiero@ncis.org )

National Coalition of Independent Scholars

\section{Research Article}

Keywords: SARS-CoV-2, COVID-19, vaccine, proteolysis, chondroitin sulfate, heparin, thrombosis, thrombocytopenia, platelets

Posted Date: December 15th, 2021

DOl: https://doi.org/10.21203/rs.3.rs-711871/v2

License: (c) (i) This work is licensed under a Creative Commons Attribution 4.0 International License. Read Full License

Version of Record: A version of this preprint was published at American Journal of Immunology on January 1st, 2021. See the published version at https://doi.org/10.3844/ajisp.2021.40.46. 


\title{
RELEASE OF ENDOGENOUS CHONDROITIN SULFATE AND HEPARIN AS CONSEQUENCE OF DYSREGULATED PROTEOLYSIS IN COVID-19
}

\author{
Marco Ruggiero \\ National Coalition of Independent Scholars
}

\begin{abstract}
Infection by severe acute respiratory syndrome-related coronavirus 2 (SARSCoV-2), the pathogen responsible for COVID-19, is associated with immune-mediated responses that lead to dysregulated activation of proteolytic enzymes; these contribute to damage to the endothelium, thrombosis, hypercoagulability, and other hematologic complications that include thrombotic thrombocytopenia, a complication of severe COVID-19 as well as a potentially fatal adverse effect of COVID-19 vaccination. The aim of this study is to demonstrate the hypothesis that thrombotic thrombocytopenia is associated with, and possibly due, to proteolysis of human plasma proteins leading to sequential release of endogenous glycosaminoglycans (GAGs), first chondroitin sulfate (CS), followed by heparin (HP). This hypothesis is based on experiments on healthy human plasma performed at the Department of Experimental Pathology and Oncology, University of Florence, Italy. The results of those experiments are here reported and reinterpreted at the light of the complications of COVID-19. Based on those results, it is hypothesized that the extension and degree of what is called "proteolytic storm" in COVID-19 determines whether only one endogenous type of GAGs (CS), or both (CS and HP), are released. Sulfated GAGs such as CS and HP exert a protective role against SARS-CoV-2 infection. However, sustained and excessive release of endogenous HP may be responsible for thrombotic thrombocytopenia just as it happens in HP-induced thrombocytopenia (HIT) a well-known side effect of HP administration that results in thromboembolisms in atypical sites, thrombocytopenia, and synthesis of autoantibodies directed against platelet factor 4 (PF4) that contribute to platelet aggregation. It is concluded that release of endogenous HP as consequence of dysregulated proteolysis occurring during COVID-19 or COVID-19 vaccination may play a fundamental role in the pathophysiology of the disease as well as in adverse reactions to vaccination.
\end{abstract}

Keywords SARS-CoV-2; COVID-19; vaccine; proteolysis; chondroitin sulfate; heparin; thrombosis; thrombocytopenia; platelets 


\section{Introduction}

Hematologic complications of COVID-19, the disease caused by severe acute respiratory syndrome-related coronavirus 2 (SARS-CoV-2), are of prognostic significance and have been associated with increased mortality. These include blood count alterations such as lymphopenia, leukopenia, thrombocytopenia, neutrophilia; hyperferritinemia and elevated lactate dehydrogenase; elevated D-dimer, fibrin degradation products, and fibrinogen; and alterations of coagulation such as disseminated intravascular coagulation, thrombosis, hypercoagulability, and thrombotic thrombocytopenia (Mina et al., 2020; Castro et al., 2021).

One particular hematologic complication, thrombotic thrombocytopenia, is observed in severely ill COVID-19 patients or, alternatively, in medically stable subjects with no anamnesis of preexisting pro-thrombotic condition, who had received COVID-19 vaccines (Dotan and Shoenfeld, 2021; Aleem et al., 2021). It has been hypothesized that thrombotic thrombocytopenia occurring in severe COVID-19, or as a side effect of vaccination, is caused by immune system dysregulation with production of autoantibodies against platelet factor 4 (PF4). This contributes to platelet aggregation and thrombosis in atypical sites such as the cerebral venous system (Ciccone, 2021).

It has been observed that the thrombotic thrombocytopenia occurring in severe COVID19 , or as a side effect of vaccination, is superimposable to the same phenomenon that may occur, as a known adverse effect, following heparin (HP) administration (HP-induced thrombocytopenia or HIT), even though the majority of COVID 19 patients or vaccinated subjects presenting this complication had not been previously exposed to HP (Dotan and Shoenfeld, 2021). In addition to the pro-aggregating pathway pertaining to PF4, it is worth noticing that HP directly activates platelets through stimulation of inositol lipid metabolism (Ruggiero et al., 1984). Direct HP-induced platelet activation occurs independently of PF4, and correlates with lipoprotein lipase activity of HP sub-fractions, but not with their anti-coagulant activity (Ruggiero et al., 1984).

A recent article (Fornasari, 2021), hypothesizes that severe COVID-19 is associated with excessive, unbalanced, sustained production and release of proteolytic enzymes, a "proteolytic 
storm" that is the main cause of immune-mediated thrombosis, hypercoagulability and the cytokine storm that, in turn, is associated with high mortality (Hojyo et al., 2020). The proteolytic storm in severe COVID-19 is thought to be caused by enzymes produced and released by recruited neutrophils; these would be responsible for unregulated activation of the four major proteolytic cascades at work in human plasma, that are the blood coagulation cascade, the complement system, the cascade of fibrinolysis and the plasma kallikrein-kinin cascade (Fornasari, 2021). The hypothesis of a proteolytic storm as a major pathogenetic factor is corroborated by the observation that non-specific, broad-spectrum protease inhibitors are being considered as effective therapeutics for COVID-19 (Sagawa et al., 2020).

The aim of this study is to demonstrate the hypothesis that thrombotic thrombocytopenia is associated with, and possibly due to, proteolysis of human plasma proteins leading to sequential release of endogenous glycosaminoglycans (GAGs), first chondroitin sulfate (CS), followed by HP. This hypothesis is based on experiments on healthy human plasma performed at the Department of Experimental Pathology and Oncology, University of Florence, Italy (Ruggiero et al., 2002). The results of those experiments are here reported and reinterpreted at the light of the complications of COVID-19. According to this hypothesis, and on the basis of our previous observations (Ruggiero et al., 2002), proteolysis of plasma proteins, as it is may occur in COVID-19, leads to release of endogenous GAGs such as CS and HP. Based on our previous observations (Ruggiero et al., 2002), it is conceivable that moderate proteolysis releases only CS, a GAG that may have a protective effect against SARS-CoV-2 entry (Zunaid et al., 2020; Song et al. 2020); however, sustained and unregulated proteolysis, the proteolytic storm, releases also endogenous HP that directly activates platelets and contributes to the thrombotic thrombocytopenia that is observed in severe COVID-19 or, as a potentially fatal adverse effect of vaccination (Dotan and Shoenfeld, 2021).

\section{Materials and Methods}

All procedures and results described in this study were performed at the Department of Experimental Pathology and Oncology of the University of Firenze, Italy (Ruggiero et al., 2002), and are here reported, explained and reinterpreted at the light of their significance in the context 
of thrombotic thrombocytopenia as it may occur in COVID-19 or COVID-19 vaccination. Figs. 1 and 2 are reproduced with permission from Pathophysiology of Haemostasis and Thrombosis, S. Karger AG, Basel, Switzerland.

\section{Materials}

Standards. The HP, used as a standard, (internal Opocrin code, EP 756; average molecular mass $12.9 \mathrm{kD}$ ) was extracted from bovine intestinal mucosa, and was kindly provided by Opocrin Research Laboratories (Modena, Italy). Chondroitin sulfate A, used as standard, was purchased from Sigma Chem. Co. (St. Louis, MO, USA).

Proteases. The proteases used for proteolysis of plasma proteins were:

Papain (EC 3.4.22.2) (Calbiochem, La Jolla, CA, USA)

Trypsin (EC 3.4.21.4) (Sigma Chem. Co., St. Louis, MO, USA)

Chymotrypsin (EC 3.4.21.1) (Sigma Chem. Co., St. Louis, MO, USA)

Pepsin (EC 3.4.23.1) (Boehringer Mannheim, Germany)

Other reagents. Chondroitinase AC (EC 4.2.2.5) and chondroitinase ABC (EC 4.2.2.4) were purchased from Sigma Chem. Co., St. Louis, MO, USA. Diethylaminoethyl (DEAE)-Sephacel was from Pharmacia (Uppsala, Sweden). Titan III Zip Zone cellulose acetate gels were from Helena Laboratories (Beaumont, TX, USA). Reagents for SDS-PAGE were from Bio-Rad (Hercules, CA, USA). Guanidine hydrochloride and other common reagents were from Sigma Chem. Co., St. Louis, MO, USA. Alcian blue was purchased from Fisher Scientific Co. (Pittsburgh, PA, USA).

\section{Methods}

The original procedure (Ruggiero et al., 2002) to release CS from its association with plasma proteins is described below. Plasma from healthy subjects who had not received any treatment with HP for at least one year, was subjected to proteolysis by sequential addition of broad-spectrum proteases in the following order: papain, trypsin, chymotrypsin, collagenase, and pepsin, each at a final concentration of $1 \mathrm{mg} / \mathrm{ml}$. Each protease was added for $24 \mathrm{~h}$ at $37^{\circ} \mathrm{C}$. With the exception of the first proteolytic step with papain, the samples were boiled before addition of 
each protease in order to inactivate the previous enzyme. At the end of the proteolytic process, centrifugation at 15,000 g for 10 min was performed in order to obtain a clear supernatant that was then stored at $-80^{\circ} \mathrm{C}$ for further processing and analyses. The supernatant was treated with $66 \%$ ethanol in order to precipitate the endogenous GAGs that are present in human plasma and were associated with plasma proteins before proteolysis. The pellet obtained by precipitation was dissolved in distilled water and dialyzed against Tris- $\mathrm{HCl}(0.05 \mathrm{M})$, at neutral $\mathrm{pH}$, in order to remove salts that would have hampered the following electrophoretic analyses. A DEAESephacel column $(1 \times 3 \mathrm{~cm})$ equilibrated in Tris- $\mathrm{HCl}(0.05 \mathrm{M})$, at neutral $\mathrm{pH}$, was used to separate plasmatic GAGs based on their affinity for the ion exchange chromatographic resin. After washing the column with $100 \mathrm{ml}$ of $0.1 \mathrm{M} \mathrm{NaCl}$, elution was performed stepwise with increasing concentrations of guanidine hydrochloride starting from $0.2 \mathrm{M}$ as described in Ruggiero et al. (2001). Guanidine salts were used instead of $\mathrm{NaCl}$ because it had been demonstrated that they represent an improvement over existing elution methods to recover GAGs from biological samples. The fractions eluted from the column of DEAE-Sephacel were lyophilized in order to reduce their volume for further analyses; following lyophilization, the fractions were dissolved in distilled water in order to reach a concentration of guanidine hydrochloride of $4 \mathrm{M}$. Following this step, they were precipitated with 2 volumes of absolute ethanol. The fractions obtained by precipitation were analyzed by cellulose acetate electrophoresis at acidic pH (1.0) as described by Cavari and Vannucchi (1993). Standard HP (Heparin EP 756) and chondroitin sulfate A were used as standards. This procedure led to release of endogenous CS but not HP (Fig. 1).

The original procedure to release HP from its association with plasma protein was identical to that described for CS up until the passage of ion exchange chromatography with DEAE-Sephacel column. In other words, the samples were subjected to the same sequence of proteolysis, centrifugation, precipitation with $66 \%$ ethanol and dialysis but, instead of being applied to the DEAE-Sephacel column, they were subjected to an original and counterintuitive procedure of electrophoresis overrun. That is, they were subjected to SDS-PAGE using a high concentration (15\%) of acrylamide and operating under reducing conditions that consisted in the presence of dithiothreitol at $0.15 \%$ concentration. The electrophoresis was overrun with the goal of entrapping proteins and peptides - still associated with HP and protected from proteolytic 
degradation by their association with HP - inside the polyacrylamide gel at the same time letting HP escape from the gel and entering the buffer used for the electrophoresis. This was achieved as a result of the inherent flexibility of the HP chain that prevented its entrapment inside the polyacrylamide gel at variance with proteins and peptides that could not exit the molecular sieve constituted by the $15 \%$ polyacrylamide gel under reducing conditions. In other words, the electrophoresis was run for a period of time significantly longer than that normally utilized to separate proteins and peptides. Standard HP and protein molecular weight markers were used to assess duration of the electrophoresis; the overrun was halted when all the HP standard had disappeared from the gel while the protein molecular weight markers, although spread, were still entrapped inside the gel. In this manner, HP, free of its association with plasma proteins or peptides, accumulated in the buffer of the electrophoresis. The buffer was then collected and subjected to ion exchange chromatography with guanidine salts, ethanol precipitation and cellulose acetate electrophoresis at $\mathrm{pH} 1.0$ exactly as with the procedure to evidence CS. By adding the step constituted by SDS-PAGE overrun, HP was released from its association with plasma proteins and peptides and could be evidenced by cellulose acetate electrophoresis (Fig. 2).

\section{Results}

Fig. 1, reproduced with permission from Pathophysiology of Haemostasis and Thrombosis, S. Karger AG, Basel, Switzerland (Ruggiero et al., 2002), demonstrates that endogenous CS can be recovered after sequential proteolysis of plasma, but not endogenous HP, since no material positive to Alcian blue and co-migrating with standard HP was evidenced by cellulose acetate electrophoresis. Absence of HP recovery was confirmed by absence of anticoagulant activity in the fractions eluted from the DEAE-Sephacel column following plasma proteolysis. CS A, used as standard (Fig. 1, lane C) allowed recognition of bands that represent CS with different degree of sulfation (Staprans and Felts, 1985), and were sensitive to chondroitinase $\mathrm{AC}$ and $\mathrm{ABC}$, but not to nitrous acid. In the context of COVID-19, it can be hypothesized that CS is released from its association with plasma proteins and peptides by immune response-mediated proteolysis; this might represent a defensive or protective response (Song et al., 2020) since CS, by cause of the high density of negative charges, may block the 
entry of SARS-CoV-2 into target cells by interacting with the positively charged residues of the virus and, in particular, the TNGTKR, HKNNKS, RSYLTPGDSSSG, and QTNSPRRA sequences (Zunaid et al., 2020) that are characterized by hydrophobic residues intercalated in a sequence of positively charged residues (Jaimes et al., 2020). In addition to a direct effect on SARS-CoV-2, release of endogenous CS represents a protective factor by cause of the known anti-inflammatory (Ronca et al., 1998) and immune-modulating (Zhou et al., 2010) properties of CS that might buffer the immune responses to the viral infection, thus reducing the severity of the disease. It is worth noticing that the immune modulating effects of CS are ascribed not only to bona fide CS but also to its cleavage disaccharide products (Zhou et al., 2010) and, as demonstrated in Figs. 1 and 2, human plasma appears to contain CS cleavage products that are released following proteolysis. Calculations performed on the endogenous CS recovered following proteolysis, indicate that its concentration in human plasma is around $1 \mathrm{ug} / \mathrm{mL}$ that is an amount compatible with the anti-viral effects described by Song et al. (2020).

Fig. 2, reproduced with permission from Pathophysiology of Haemostasis and Thrombosis, S. Karger AG, Basel, Switzerland (Ruggiero et al., 2002), shows that endogenous HP can be recovered only when the additional purification step constituted by SDS-PAGE overrun is performed with the goal of freeing HP from its association with plasma proteins. Calculations, performed by densitometric comparison with standard HP, indicate that the concentration of endogenous HP in human plasma is around $7-10 \mathrm{ug} / \mathrm{mL}$ that is a concentration about 10 fold higher than that of endogenous CS. Considering that 1 unit of pure, pharmacological grade, HP corresponds to $0.002 \mathrm{mg}$, it can be inferred that the amount of endogenous HP is far greater than that required to render blood non coagulable and this is probably one of the reasons why endogenous HP is so strictly associated with plasma proteins and peptides. However, the presence of such a large amount of endogenous HP in human plasma might explain why, in exceptional circumstances such as severe COVID-19 or dysregulated immune responses to vaccination, endogenous HP may contribute to thrombotic thrombocytopenia exactly as if exogenous HP had been administered. In other words, the results shown in Fig. 2 support the hypothesis that the thrombotic thrombocytopenia observed in severe COVID-19 and, as an adverse event, following vaccination, may be due to the release of 
endogenous HP from its association with plasma proteins as consequence of dysregulated proteolytic activity.

\section{Discussion}

The present study does not elucidate the nature of the exceptional circumstances that lead to endogenous HP release with catastrophic consequences, even though some hypotheses can be put forward on the basis of the results presented in Fig. 2. According to this model, endogenous HP is complexed with two categories of plasma proteins; those that are hydrolyzed by sequential addition of proteases in a manner analogous to what observed for CS; and those that are protected by proteases as a result of their association with HP, and are removed only by the SDSPAGE overrun. While it is easy to presume that the proteolytic storm associated with COVID19 , or with vaccination, may resemble the action of the proteases described in this study, it is more difficult to imagine which molecular mechanisms are mimicked by the SDS-PAGE overrun that is, essentially, an electrostatic stripping of endogenous HP. A hint may come from the observation that endogenous HP is complexed also with phospholipids, notably phosphatidylcholine, as a results of the interaction between the quaternary ammonium group of phosphatidylcholine and the sulfate groups of HP (Vannucchi et al., 1985). Such an interaction between endogenous HP and phosphatidylcholine occurs at the level of the plasma membrane and might represent the physiologic mimicry of the SDS-PAGE overrun. In this case, moderate proteolysis occurring in COVID-19 or following vaccination, may be sufficient to release endogenous CS, possibly with protective effects, but would not be sufficient to release endogenous HP exactly as it happens in the procedure leading to the results depicted in Fig. 1. If, however, proteolysis persists or goes unbalanced, the amount of endogenous HP, still bound to plasma proteins and peptides, increases up to the point that some HP molecules interact with the polar head of phosphatidylcholine at the level of plasma membranes and this electrostatic interaction frees endogenous HP from its bonds with proteins and peptides as if it were a SDSPAGE overrun. This hypothesis is corroborated by the observation that HP does indeed interact with phospholipid bilayers with disruptive effects such as red blood cell hemolysis (Patel et al., 1983). As far as the mechanism of action involved in endogenous HP-induced thrombotic thrombocytopenia is concerned, it is worth considering a direct effect of HP on platelet plasma 
membrane with activation of inositol lipid metabolism (Ruggiero et al., 1984), and formation of calcium-mobilizing inositol 1,4,5-trisphosphate and protein kinase C-activating 1,2-

diacylglycerol (Watson et al., 1986). This mechanism is complementary to that pertaining to PF4 involvement. Furthermore, it has been demonstrated that platelets contain endogenous HP that is released following platelet adhesion (Vannucchi et al., 1982). Therefore, platelets stimulated by endogenous HP, either directly or with the intermediation of PF4, release additional HP that leads to the catastrophic thrombotic thrombocytopenia observed in severe COVID-19 or following vaccination.

\section{Limitations and future directions}

The first limitation of this study is that it is based on experiments performed on human plasma from healthy subjects where extensive proteolysis was achieved in vitro with an array of proteases whose enzymatic mechanism of action does not necessarily reflect the pattern of proteolysis of plasma proteins observed in the proteolytic storm associated with COVID-19 or with vaccination. Another limitation concerns the fact that the mechanisms responsible for separating endogenous HP from those plasma proteins that are protected from the enzymatic action of proteases as a result of their association with the GAG is not known. In particular, it is not known whether the mechanism of action here proposed - binding of endogenous HP to phosphatidylcholine - is at work in COVID-19 even though it is known that SARS-CoV and MERS-CoV can remodel the lipidome of the infected cells (Pimentel and Rodríguez-Alcalá, 2021).

Future directions in this field might include determining the profiles of endogenous plasma GAGs in COVID-19 patients experiencing thrombotic thrombocytopenia and compare their profiles with those of healthy subjects and of COVID-19 patients without complications. Other future directions might include the study of endogenous plasma GAGs in COVID-19 vaccinated subjects experiencing thrombotic thrombocytopenia and compare their profiles with those of vaccinated subjects with no complications as well as with those of unvaccinated subjects. 


\section{Acknowledgements}

The Author wishes to thank Dr. Simonetta Vannucchi whose contribution to the field of endogenous GAGs since 1977 has been priceless although little acknowledged.

\section{Disclosures}

Marco Ruggiero is the founder of Silver Spring Sagl, a company producing supplements and probiotics including some containing CS. He served as CEO of the company until his retirement in 2020. None of the products invented by M. Ruggiero or commercialized by Silver Spring Sagl is mentioned in this article. Marco Ruggiero is member of the Editorial Board of the American Journal of Immunology and is waived from the Article Processing fee for this contribution; he receives no remuneration for his editorial work.

\section{Advisory}

No information in this study is intended or implied to be a substitute for professional medical advice, diagnosis or treatment.

\section{Ethics}

This article is original and contains material that has not been submitted or published in any scientific journal. A pre-print of the first submission of this article had been posted in Research Square (doi: 10.21203/rs.3.rs-711871/v1; https://www.researchsquare.com/article/rs-711871/v1). The current article, however, after thorough revision following the Reviewers' comments, is significantly different from the first version of the pre-print. 


\section{References}

Aleem A, Nadeem AJ. Coronavirus (COVID-19) Vaccine-Induced Immune Thrombotic Thrombocytopenia (VITT). 2021 Apr 25. In: StatPearls [Internet]. Treasure Island (FL): StatPearls Publishing; 2021 Jan-. PMID: 34033367.

Castro RA, Frishman WH. Thrombotic Complications of COVID-19 Infection: A Review. Cardiol Rev. 2021 Jan/Feb;29(1):43-47. doi: 10.1097/CRD.0000000000000347. PMID: 32947478 .

Cavari S, Vannucchi S. Glycosaminoglycans exposed on the endothelial cell surface. Binding of heparin-like molecules derived from serum. FEBS Lett. 1993 May 24;323(1-2):155-8. doi: 10.1016/0014-5793(93)81469-g. PMID: 8495730.

Ciccone A. SARS-CoV-2 vaccine-induced cerebral venous thrombosis. Eur J Intern Med. 2021 May 25:S0953-6205(21)00185-0. doi: 10.1016/j.ejim.2021.05.026. Epub ahead of print. PMID: 34090750 ; PMCID: PMC8148433.

Dotan A, Shoenfeld Y. Perspectives on vaccine induced thrombotic thrombocytopenia. J Autoimmun. 2021 Jul;121:102663. doi: 10.1016/j.jaut.2021.102663. Epub 2021 May 18. PMID: 34020254; PMCID: PMC8129886.

Fornasari PM. SARS-CoV-2 and Proteolytic Storm: Neutrophils and Proteolytic Enzymes are the "Prima Donna" in Severe COVID-19. Serine Proteases Inhibitors are Valuable Treatment Options. Am J Blood Rev. 2021; 1(1): 1001. https://www.remedypublications.com/americanjournal-of-blood-reviews-abstract.php?aid=6745

Hojyo S, Uchida M, Tanaka K, Hasebe R, Tanaka Y, Murakami M, Hirano T. How COVID-19 induces cytokine storm with high mortality. Inflamm Regen. 2020 Oct 1;40:37. doi:

10.1186/s41232-020-00146-3. PMID: 33014208; PMCID: PMC7527296.

Jaimes JA, Andre NM, Millet JK, Whittaker GR. Structural modeling of 2019-novel coronavirus $(\mathrm{nCoV})$ spike protein reveals a proteolytically-sensitive activation loop as a distinguishing feature compared to SARS-CoV and related SARS-like coronaviruses. arXiv:2002.06196. eprint arXiv:2002.06196. https://arxiv.org/abs/2002.06196 https://arxiv.org/pdf/2002.06196.pdf

Mina A, van Besien K, Platanias LC. Hematological manifestations of COVID-19. Leuk Lymphoma. 2020 Dec;61(12):2790-2798. doi: 10.1080/10428194.2020.1788017. Epub $2020 \mathrm{Jul}$ 9. PMID: 32643489.

Patel HM, Parvez N, Field J, Ryman BE. Lytic effect of heparin on liposomes: possible mechanism of lysis of red blood cells by heparin. Biosci Rep. 1983 Jan;3(1):39-46. doi: 10.1007/BF01121569. PMID: 6839010. 
Pimentel LL, Rodríguez-Alcalá LM. Cholesterol, inflammation, and phospholipids: COVID-19 share traits with cardiovascular disease. Biochim Biophys Acta Mol Cell Biol Lipids.

2021;1866(1):158839. doi:10.1016/j.bbalip.2020.158839

Ronca F, Palmieri L, Panicucci P, Ronca G. Anti-inflammatory activity of chondroitin sulfate. Osteoarthritis Cartilage. 1998 May;6 Suppl A:14-21. doi: 10.1016/s1063-4584(98)80006-X. PMID: 9743814.

Ruggiero M, Caldini R, Chevanne M, Melli M, Pacini S, Gulisano M, Vannucchi S. A method for separation of heparin species from biological samples by ethanol precipitation of compounds solubilized in guanidine hydrochloride. Ital J Anat Embryol. 2001 Jan-Mar;106(1):35-46. PMID: 11410997.

Ruggiero M, Fedi S, Bianchini P, Vannucchi S, Chiarugi V. Molecular events involved in the proaggregating effect of heparin on human platelets. Biochim Biophys Acta. 1984 Nov 28;802(2):372-7. doi: 10.1016/0304-4165(84)90185-5. PMID: 6498225.

Ruggiero M, Melli M, Parma B, Bianchini P, Vannucchi S. Isolation of endogenous anticoagulant N-sulfated glycosaminoglycans in human plasma from healthy subjects. Pathophysiol Haemost Thromb. 2002 Jan-Feb;32(1):44-9. doi: 10.1159/000057288. PMID: 12214163.

Sagawa T, Inoue KI, Takano H. Use of protease inhibitors for the prevention of COVID-19. Prev Med. 2020 Dec;141:106280. doi: 10.1016/j.ypmed.2020.106280. Epub 2020 Oct 6. PMID: 33035549 ; PMCID: PMC7537597.

Song S, Peng H, Wang Q, Liu Z, Dong X, Wen C, Ai C, Zhang Y, Wang Z, Zhu B. Inhibitory activities of marine sulfated polysaccharides against SARS-CoV-2. Food Funct. 2020 Sep 23;11(9):7415-7420. doi: 10.1039/d0fo02017f. PMID: 32966484.

Staprans I, Felts JM. Isolation and characterization of glycosaminoglycans in human plasma. J Clin Invest. 1985 Nov;76(5):1984-91. doi: 10.1172/JCI112198. PMID: 4056061; PMCID: PMC424260.

Vannucchi S, Fibbi G, Pasquali F, Del Rosso M, Cappelletti R, Chiarugi V. Adhesion-dependent heparin production by platelets. Nature. 1982 Mar 25;296(5855):352-3. doi: 10.1038/296352a0. PMID: 7063033.

Vannucchi S, Ruggiero M, Chiarugi V. Complexing of heparin with phosphatidylcholine. A possible supramolecular assembly of plasma heparin. Biochem J. 1985 Apr 1;227(1):57-65. doi: 10.1042/bj2270057. PMID: 3994692; PMCID: PMC1144808.

Watson SP, Ruggiero M, Abrahams SL, Lapetina EG. Inositol 1,4,5-trisphosphate induces aggregation and release of 5-hydroxytryptamine from saponin-permeabilized human platelets. J Biol Chem. 1986 Apr 25;261(12):5368-72. PMID: 3082884. 
Zhou J, Nagarkatti P, Zhong Y, Nagarkatti M. Immune modulation by chondroitin sulfate and its degraded disaccharide product in the development of an experimental model of multiple sclerosis. J Neuroimmunol. 2010 Jun;223(1-2):55-64. doi: 10.1016/j.jneuroim.2010.04.002. PMID: 20434781; PMCID: PMC4138964.

Zunaid IR, Pacini S, Ruggiero M. Significance of hydrophobic and charged sequence similarities in sodium-bile acid cotransporter and vitamin D-binding protein macrophage activating factor. bioRxiv 2020. doi: https://doi.org/10.1101/2020.03.03.975524 


\section{Figure legends}

Figure 1. Cellulose acetate electrophoresis of fractions eluted from DEAE-Sephacel. $\mathrm{H}$ : heparin standard $(12.9 \mathrm{kD})$. C: chondroitin sulfate A. Lane 1: elution at $0.2 \mathrm{M}$ guanidine hydrochloride. Lane 2: elution at $0.3 \mathrm{M}$ guanidine hydrochloride. Lane 3: elution at $0.4 \mathrm{M}$ guanidine hydrochloride. Lane 4: elution at $0.6 \mathrm{M}$ guanidine hydrochloride. Lane 5: elution at 1 M guanidine hydrochloride. Lane 6: elution at $2 \mathrm{M}$ guanidine hydrochloride. Each aliquot loaded onto the cellulose acetate gel $(2 \mathrm{uL})$ was the equivalent of $1.0 \mathrm{~mL}$ of the initial plasma sample. Therefore, the amount of glycosaminoglycans separated by ion exchange chromatography, evaluated by comparison with the standards $(200 \mathrm{ug} / \mathrm{mL})$, was $1.0 \mathrm{ug} / \mathrm{mL}$.

Figure 2. Cellulose acetate electrophoresis of fractions eluted from DEAE-Sephacel after having been subjected to SDS-PAGE overrun.

A. H: heparin standard $(12.9 \mathrm{kD})$. C: chondroitin sulfate A. Lane 1: elution at $0.2 \mathrm{M}$ guanidine hydrochloride. Lane 2: elution at $0.3 \mathrm{M}$ guanidine hydrochloride. Lane 3: elution at $0.4 \mathrm{M}$ guanidine hydrochloride. Lane 4: elution at $0.6 \mathrm{M}$ guanidine hydrochloride. Lane 5: elution at 1 M guanidine hydrochloride. Lane 6: elution at $2 \mathrm{M}$ guanidine hydrochloride. B. The same fractions treated with nitrous acid. The amount of nitrous acid-sensitive glycosaminoglycans, that is endogenous HP, calculated as above, was 7-10 ug/mL. 
Figure 1

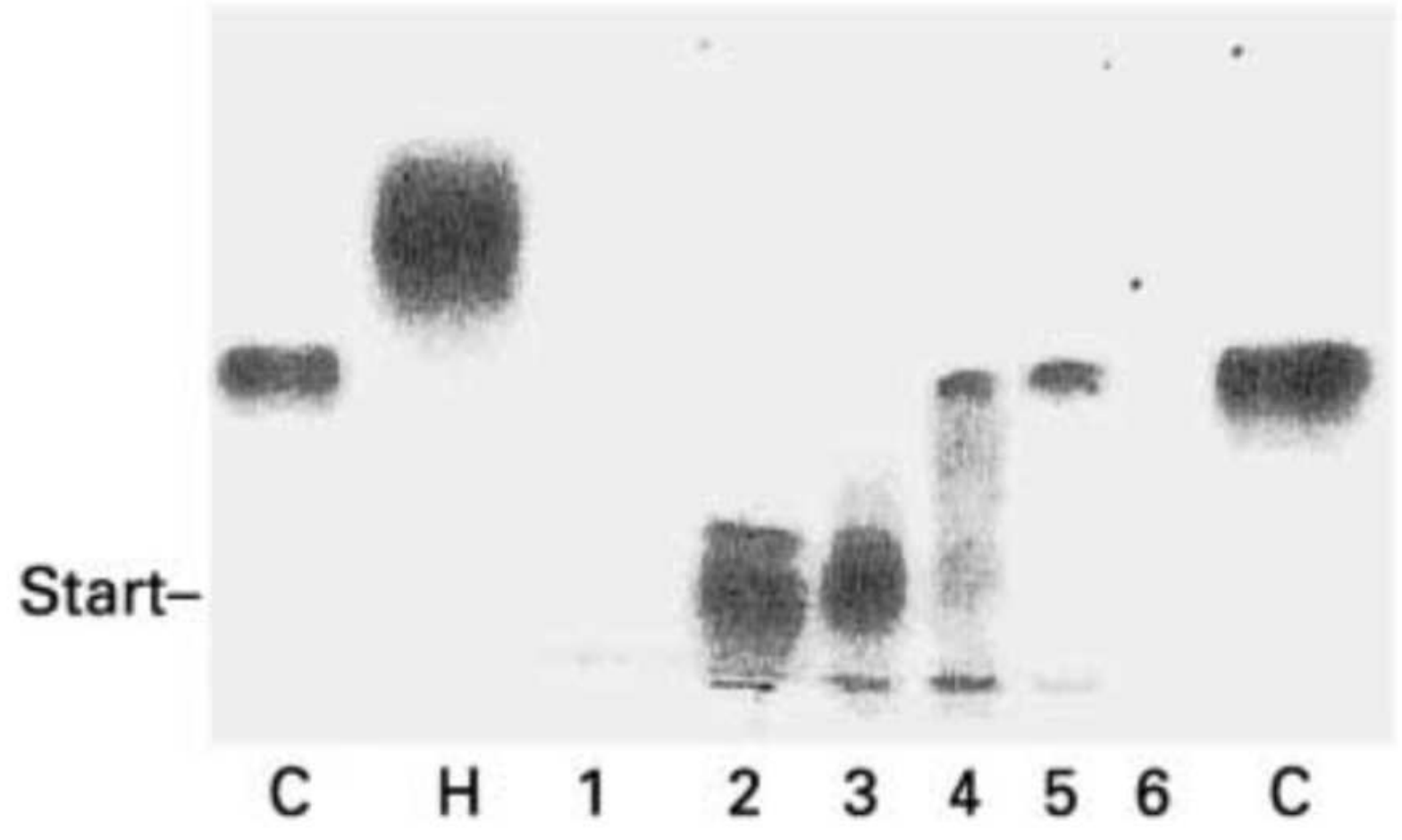


Figure 2
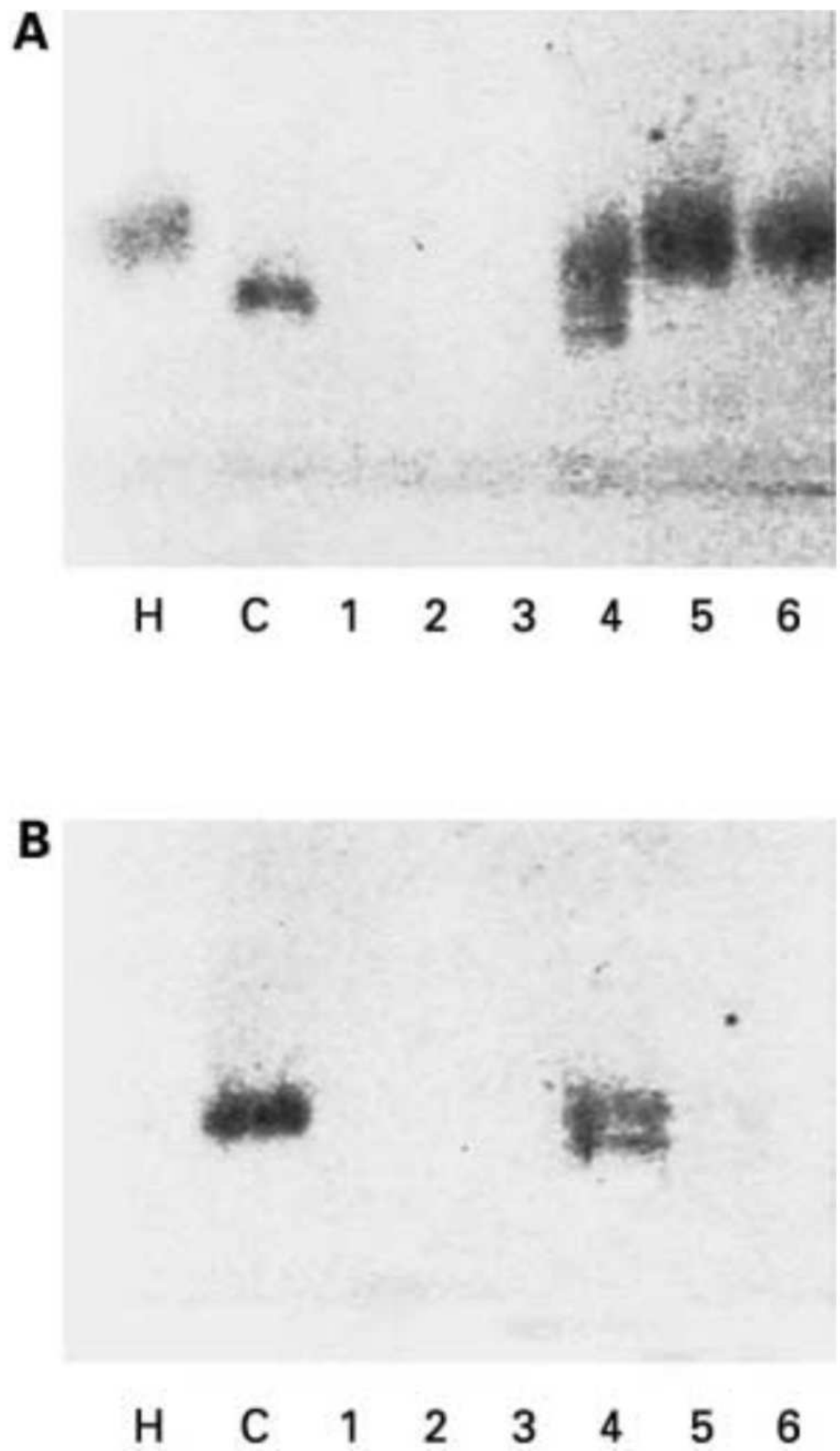